
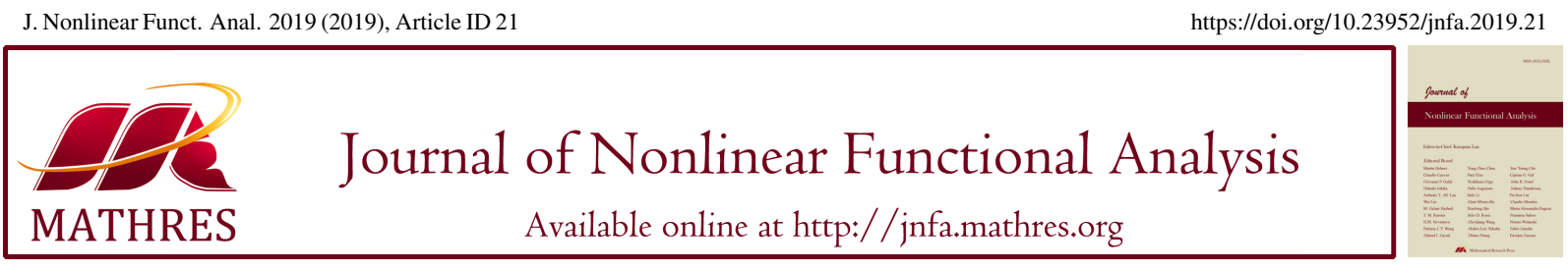

\title{
RESONANT FRACTIONAL DIFFERENTIAL EQUATIONS WITH MULTI-POINT BOUNDARY CONDITIONS ON $(0,+\infty)$
}

\author{
SMAÏL DJEBALI ${ }^{1,3, *}$, ABDELLATIF GHENDIR AOUN ${ }^{2,3}$ \\ ${ }^{1}$ Department of Mathematics and Statistics, College of Sciences, \\ Imam Mohammad Ibn Saud Islamic University, P.O. Box 90950, Riyadh 11623, Saudi Arabia \\ ${ }^{2}$ Department of Mathematics, Faculty of Exact Sciences, Hamma Lakhdar University, 39000 El-Oued, Algeria \\ ${ }^{3}$ Laboratory Theory of Fixed Point and Applications, ENS, BP 92 Kouba, Algiers, 16016, Algeria
}

\begin{abstract}
The aim of this paper is to establish the existence of solutions for a class of multi-point boundary value problems for a fractional differential equation at resonance which is posed on the positive half-line. The arguments we have used are mainly based on Mawhin's coincidence degree theory. An example is provided to illustrate our main existence result.

Keywords. Coincidence degree theory; Fractional differential equation; Infinite interval; Multi-point boundary value problem; Resonance.
\end{abstract}

2010 Mathematics Subject Classification. 34B10, 34B40, 34F15.

\section{INTRODUCTION}

Various real-life phenomena are described by fractional differential equations (FDEs for brevity). FDEs occur in many fields such as physics, chemistry, engineering, and control of dynamical systems, etc. During the last few decades, many results have been devoted to fractional calculus and fractional differential equations. Recently, boundary value problems (BVPs for brevity) for differential equations of integer orders at resonance have been studied by many authors; see, for example, $[1,2,3,4]$ and the references therein. However, in the resonance case, only few results on the existence of solutions of FDEs on unbounded domains were established. In the latter case, problems are approached in several ways. A classical method consists in decomposing the space as a direct sum of subspaces. One is the kernel of a suitable operator, and then we can consider the corresponding projections on these spaces.

In the recent literature, we can find some types of multi-point boundary value problems associated with fractional differential equations at resonance on bounded domains; see, for example, $[5,6,7,8,9,10]$ and the references therein. Let us describe some of them. Bai [5] considered the following $m$-point

${ }^{*}$ Corresponding author.

E-mail addresses: djebali@ hotmail.com (S. Djebali), ghendirmaths@gmail.com (A. Ghendir Aoun).

Received November 28, 2018; Accepted April 30, 2019.

(C)2019 Journal of Nonlinear Functional Analysis 
fractional $\mathrm{BVP}$ on $[0,1]$ in the resonance case:

$$
\left\{\begin{array}{l}
D_{0^{+}}^{\alpha} u(t)=f\left(t, u(t), D_{0^{+}}^{\alpha-1} u(t)\right)+e(t), \quad t \in(0,1), \\
I_{0^{+}}^{2-\alpha} u(0)=0, \quad u(1)=\sum_{i=1}^{m-2} \beta_{i} u\left(\eta_{i}\right)
\end{array}\right.
$$

where $1<\alpha \leq 2$ is a real number, $f:[0,1] \times \mathbb{R} \times \mathbb{R} \rightarrow \mathbb{R}$ is a continuous function and $e \in L^{1}[0,1]$, $\beta_{i} \in \mathbb{R}, i=1,2, \ldots, m-2,0<\eta_{1}<\eta_{2}<\ldots<\eta_{m-2}<1$ are given constants such that $\sum_{i=1}^{m-2} \beta_{i} \eta_{i}^{\alpha-1}=1$. Using the coincidence degree theory, the existence of solutions was established.

In [11], Chen and Tang studied the following two point BVP of fractional differential equations on $(0,+\infty)$ in the non-resonance case:

$$
\left\{\begin{array}{l}
D_{0^{+}}^{\alpha} u(t)=f(t, u(t)), \quad t \in(0,+\infty), \\
u(0)=u^{\prime}(0)=u^{\prime \prime}(0)=0, \quad D_{0^{+}}^{\alpha-1} u(0)=\lim _{t \rightarrow+\infty} D_{0^{+}}^{\alpha-1} u(t),
\end{array}\right.
$$

where $3<\alpha<4$ and $f:[0,+\infty) \times \mathbb{R} \rightarrow \mathbb{R}$ is a continuous function and for each $l>0$, there exists $\phi_{l} \in C[0,+\infty) \cap L^{1}[0,+\infty)$ satisfying $\sup _{t \geq 0}\left|\phi_{l}(t)\right|<+\infty$ and $\phi_{l}(t)>0, t>0$ such that

$$
|u|<l \text { implies }\left|f\left(t,\left(1+t^{\alpha-1}\right) u\right)\right| \leq \phi_{l}(t), \text { a.e. } t \geq 0 .
$$

Employing the Leggett-Williams norm-type theorem, they have presented some results on the existence of positive solutions.

In [3], Jiang and Yang established the existence of positive solutions for the multi-point bvp at resonance on the half-line

$$
\left\{\begin{array}{l}
u^{\prime \prime}(t)+f(t, u(t))=0, \quad t \in(0,+\infty) \\
u(0)=0, \quad u^{\prime}(+\infty)=\sum_{i=1}^{m-1} \alpha_{i} u^{\prime}\left(\xi_{i}\right)
\end{array}\right.
$$

where $f:[0,+\infty) \times \mathbb{R} \rightarrow \mathbb{R}$ is continuous, $f(t, 0)$ is not always equal to $0, \alpha_{i}>0, \sum_{i=1}^{m-1} \alpha_{i}=1, i=$ $1,2, \ldots, m-1,0=\xi_{1}<\xi_{2}<\ldots<\xi_{m-1}<+\infty$. Their results also rely on the Leggett-Williams normtype theorem.

In [10], Zhou, Kou, and Xie studied the existence of solutions for the following fractional differential equation at resonance with three point bvp on the half-line:

$$
\left\{\begin{array}{l}
D_{0^{+}}^{\alpha} u(t)=f\left(t, u(t), D_{0^{+}}^{\alpha-1} u(t)\right), \quad t \in(0,+\infty), \\
u(0)=0, \quad \lim _{t \rightarrow+\infty} D_{0^{+}}^{\alpha-1} u(t)=\beta u(\eta),
\end{array}\right.
$$

where $1<\alpha \leq 2, \eta>0$ such that $\Gamma(\alpha)=\beta \eta^{\alpha-1}, f:[0,+\infty) \times \mathbb{R} \times \mathbb{R} \rightarrow \mathbb{R}$ is an $S$-Carathéeodory function. Their analysis is based on the Mawhin's coincidence degree.

Motivated by these works, we consider in this paper the existence of solutions for the following multipoint BVP of fractional differential equation:

$$
\left\{\begin{array}{l}
D_{0^{+}}^{\alpha} u(t)=f\left(t, u(t), D_{0^{+}}^{\alpha-1} u(t)\right), \quad t \in(0,+\infty), \\
I_{0^{+}}^{2-\alpha} u(0)=0, \quad \lim _{t \rightarrow+\infty} D_{0^{+}}^{\alpha-1} u(t)=\sum_{i=1}^{m-2} \beta_{i} D_{0^{+}}^{\alpha-1} u\left(\xi_{i}\right),
\end{array}\right.
$$

where $1<\alpha \leq 2,0<\xi_{1}<\xi_{2}<\ldots<\xi_{m-2}<+\infty, \beta_{i}>0, i=1,2, \ldots, m-2, D_{0^{+}}^{\alpha}$ refers to the standard Riemann-Liouville fractional derivative and $I_{0^{+}}^{\alpha}$ is the standard Riemann-Liouville fractional integral. 
Unlike [12], we assume

$$
\text { (H0) } \quad \sum_{i=1}^{m-2} \beta_{i}=1
$$

Thus the BVP is under resonance.

More precisely, we establish some sufficient conditions for the existence of at least one solution for BVP (1.1). Our approach is motivated by [5] but we consider here the infinite interval setting. A new and general existence result is obtained by making use of the Mawhin's coincidence degree in a suitable Banach space. The organization of this paper is as follows. Section 2 is devoted to presenting some definitions and lemmas which are crucial in our discussion. In Section 3, we prove some technical lemmas which are needed later. Section 4 is devoted to our main existence results. In Section 5, an example is provided to illustrate our theoretical results.

\section{PRELIMINARIES}

First, we recall some notations and definitions which will be used later.

Definition 2.1. Let $X$ and $Y$ be normed spaces. A linear operator $L: D(L) \subset X \rightarrow Y$ is said to be a Fredholm operator of index zero provided that

(i) $\operatorname{Im}(L)$ is a closed subset of $Y$,

(ii) $\operatorname{dim} \operatorname{Ker}(L)=\operatorname{codim} \operatorname{Im}(L)<+\infty$.

Definition 2.2. Let $X$ be a normed space. A linear operator $P: X \rightarrow X$ is said to be a projection if $P \circ P=P$. In this case, $I-P: X \rightarrow X$ is also a projection and $\operatorname{Ker}(P)=\operatorname{Im}(I-P), \operatorname{Im}(P)=\operatorname{Ker}(I-P)$.

Let $X, Y$ be real Banach spaces. Let $L: D(L) \subset X \rightarrow Y$ be a linear operator which is a Fredholm map of index zero and $P: X \rightarrow X, Q: Y \rightarrow Y$ be continuous projectors such that $\operatorname{Im}(P)=\operatorname{ker}(L), \operatorname{Ker}(Q)=$ $\operatorname{Im}(L)$, and $X=\operatorname{Ker}(L) \oplus \operatorname{Ker}(P), Y=\operatorname{Im}(L) \oplus \operatorname{Im}(Q)$. It follows that $\left.L\right|_{D(L) \cap \operatorname{Ker}(P)}: D(L) \cap \operatorname{Ker}(P) \rightarrow$ $\operatorname{Im}(L)$ is invertible, and its inverse is denoted by $K_{P}$.

Let $\Omega$ be an open bounded subset of $X$ such that $D(L) \cap \Omega \neq \emptyset$. The map $N: X \rightarrow Y$ is said to be $L$-compact on $\bar{\Omega}$ if $Q N(\bar{\Omega})$ is bounded and $K_{P}(I-Q N): \bar{\Omega} \rightarrow X$ is compact.

Theorem 2.3. [13] Let $L$ be a Fredholm operator of index zero and let $N$ be L-compact on $\bar{\Omega}$. Assume that the following conditions are satisfied:

(1) $L u \neq \lambda N u$ for every $(u, \lambda) \in[(D(L) \backslash \operatorname{Ker}(L)) \cap \partial \Omega] \times(0,1)$,

(2) $N u \notin \operatorname{Im}(L)$ for every $u \in \operatorname{Ker}(L) \cap \partial \Omega$,

(3) $\operatorname{deg}\left(\left.Q N\right|_{\operatorname{Ker}(L)}, \operatorname{Ker}(L) \cap \Omega, 0\right) \neq 0$, where $Q: Y \rightarrow Y$ is a projection such that $\operatorname{Im}(L)=\operatorname{Ker}(Q)$.

Then the equation $L u=N u$ has at least one solution in $D(L) \cap \bar{\Omega}$.

We also collect some definitions and basic lemmas from fractional calculus (see $[14,15]$ for more details). The Gamma function extends the factorial to positive real numbers (and even to complex numbers with positive real parts).

Definition 2.4. For $\alpha>0$, the Euler Gamma function is defined by

$$
\Gamma(\alpha)=\int_{0}^{+\infty} t^{\alpha-1} e^{-t} d t
$$


Definition 2.5. Let $p>0$ and $q>0$. The Euler Beta function is defined by

$$
B(p, q)=\int_{0}^{1} t^{p-1}(1-t)^{q-1} d t
$$

Proposition 2.6. Let $\alpha>0, p>0, q>0$ and $n$ a positive integer. Then

$$
\Gamma(\alpha+1)=\alpha \Gamma(\alpha), \quad \Gamma\left(n+\frac{1}{2}\right)=\frac{\sqrt{\pi} \Gamma(2 n+1)}{2^{2 n} \Gamma(n+1)}, \quad B(p, q)=\frac{\Gamma(p) \Gamma(q)}{\Gamma(p+q)} .
$$

Hence

$$
\Gamma(\alpha+n)=\alpha(\alpha+1)(\alpha+2) \ldots(\alpha+n-1) \Gamma(\alpha) .
$$

In particular

$$
\Gamma(1)=\int_{0}^{+\infty} e^{-t} d t=1, \quad \Gamma\left(\frac{1}{2}\right)=\sqrt{\pi}
$$

and

$$
\Gamma(n+1)=n !, \quad \Gamma\left(n+\frac{1}{2}\right)=\frac{\sqrt{\pi}(2 n) !}{2^{2 n} n !} .
$$

Definition 2.7. The fractional integral of order $\alpha>0$ for function $h$ is defined by

$$
I_{0^{+}}^{\alpha} h(t)=\frac{1}{\Gamma(\alpha)} \int_{0}^{t}(t-s)^{\alpha-1} h(s) d s,
$$

provided that the right-hand side is point-wise defined on $(0,+\infty)$.

Definition 2.8. For a function $h$ on the interval $[0,+\infty)$, the Riemann-Liouville fractional derivative of order $\alpha>0$ is defined by

$$
D_{0^{+}}^{\alpha} h(t)=\left(\frac{d}{d t}\right)^{n} I_{0^{+}}^{n-\alpha} h(t)=\frac{1}{\Gamma(n-\alpha)}\left(\frac{d}{d t}\right)^{n} \int_{0}^{t} \frac{h(s)}{(t-s)^{\alpha-n+1}} d s,
$$

where $n=[\alpha]+1$.

Lemma 2.9. [14] Let $\alpha>0$. Then

$$
I_{0^{+}}^{\alpha} D_{0^{+}}^{\alpha} u(t)=u(t)+c_{1} t^{\alpha-1}+c_{2} t^{\alpha-2}+\ldots+c_{n} t^{\alpha-n}
$$

for some $c_{i} \in \mathbb{R}(i=1,2, \ldots, n), n=[\alpha]+1$.

Proposition 2.10. [15] The following composition relations between fractional and integral derivatives hold:

(a) $D_{0^{+}}^{\alpha} I_{0^{+}}^{\alpha} h(t)=h(t), \quad \alpha>0, \quad h \in L^{1}[0,+\infty)$.

(b) $D_{0^{+}}^{\alpha} I_{0^{+}}^{\gamma} h(t)=I_{0^{+}}^{\gamma-\alpha} h(t), \quad \gamma>\alpha>0, \quad h \in L^{1}[0,+\infty)$.

(c) $I_{0^{+}}^{\alpha} I_{0^{+}}^{\gamma} h(t)=I_{0^{+}}^{\alpha+\gamma} h(t), \quad \alpha>0, \quad \gamma>0 \quad h \in L^{1}[0,+\infty)$.

(d) $D_{0^{+}}^{\alpha} t^{\lambda}=\frac{\Gamma(\lambda+1)}{\Gamma(\lambda-\alpha+1)} t^{\lambda-\alpha}$, for $\lambda>-1$, in particular for $D_{0^{+}}^{\alpha} t^{\alpha-m}=0$,

$m=1,2, \ldots, N$, where $N$ is the smallest integer greater than or equal to $\alpha$.

(e) $I_{0^{+}}^{\alpha} t^{\lambda}=\frac{\Gamma(\lambda+1)}{\Gamma(\alpha+\lambda+1)} t^{\alpha+\lambda}, \quad \alpha>0, \quad \lambda>-1$.

(f) If $1 \leq \alpha \leq 2$, then $\frac{1}{\Gamma(\alpha)} \geq 1$. 


\section{RELATED LEMMAS}

Consider the Banach space $X$ defined by

$$
X=\left\{u: u, D_{0^{+}}^{\alpha-1} u \in C([0,+\infty), \mathbb{R}): \lim _{t \rightarrow+\infty} e^{-t}|u(t)| \text { and } \lim _{t \rightarrow+\infty} e^{-t}\left|D_{0^{+}}^{\alpha-1} u(t)\right| \text { exist }\right\}
$$

with the norm

$$
\|u\|_{X}=\max \left\{\sup _{t \geq 0} e^{-t}|u(t)|, \sup _{t \geq 0} e^{-t}\left|D_{0^{+}}^{\alpha-1} u(t)\right|\right\} .
$$

Let $Y=L^{1}[0,+\infty)$ be the space of Lebesgue integrable functions equipped with the norm $\|u\|_{1}=$ $\int_{0}^{+\infty}|u(t)| d t$

Define the operator $L: D(L) \subset X \rightarrow Y$ by $L u=D_{0^{+}}^{\alpha} u$, where

$$
D(L)=\left\{u \in X: I_{0^{+}}^{2-\alpha} u(0)=0, \quad \lim _{t \rightarrow+\infty} D_{0^{+}}^{\alpha-1} u(t)=\sum_{i=1}^{m-2} \beta_{i} D_{0^{+}}^{\alpha-1} u\left(\xi_{i}\right)\right\} .
$$

Let $N: X \rightarrow Y$ be the operator

$$
N u(t)=f\left(t, u(t), D_{0^{+}}^{\alpha-1} u(t)\right), t \in[0,+\infty) .
$$

Then problem (1.1) can be written as $L u=N u$.

We next list some conditions.

(H1) $f:[0,+\infty) \times \mathbb{R} \times \mathbb{R} \rightarrow \mathbb{R}$ is a continuous function.

(H2) There exist nonnegative functions $e^{t} a(t), e^{t} b(t), c(t) \in L^{1}[0,+\infty)$ with

$$
\frac{2}{\Gamma(\alpha)} \int_{0}^{+\infty} e^{s}(a(s)+b(s)) d s<1
$$

such that

$$
|f(t, u, v)| \leq a(t)|u|+b(t)|v|+c(t), \text { for all } u, v \in \mathbb{R} \text { and } t \in[0,+\infty) .
$$

(H3) There exists a constant $M_{1}>0$ such that, if $\left|D_{0^{+}}^{\alpha-1} u(t)\right|>M_{1}$ for all $t \geq 0$, then

$$
\sum_{i=1}^{m-2} \beta_{i} \int_{\xi_{i}}^{+\infty} f\left(s, u(s), D_{0^{+}}^{\alpha-1} u(s)\right) d s \neq 0 .
$$

(H4) There exists a constant $M_{2}>0$ such that, for any $u(t)=c_{0} t^{\alpha-1}$ with $\left|c_{0}\right|>M_{2}$ either

$$
c_{0} \frac{\sum_{i=1}^{m-2} \beta_{i}}{\sum_{i=1}^{m-2} \beta_{i} e^{-\xi_{i}}} \int_{\xi_{i}}^{+\infty} f\left(s, c_{0} t^{\alpha-1}, c_{0} \Gamma(\alpha)\right) d s<0
$$

or

$$
c_{0} \frac{\sum_{i=1}^{m-2} \beta_{i}}{\sum_{i=1}^{m-2} \beta_{i} e^{-\xi_{i}}} \int_{\xi_{i}}^{+\infty} f\left(s, c_{0} t^{\alpha-1}, c_{0} \Gamma(\alpha)\right) d s>0 .
$$

Since the Arzela-Ascoli theorem fails to work in space $X$, we need a modified compactness criterion.

Lemma 3.1. [16] Let $Z \subseteq X$ be a bounded set. Then $Z$ is relatively compact on $X$ if for any $u \in Z, e^{-t} u(t)$ and $e^{-t} D^{\alpha-1} u(t)$ are equicontinuous on any compact intervals of $[0,+\infty)$ and are equiconvergent at infinity. 
Definition 3.2. $e^{-t} u(t)$ and $e^{-t} D^{\alpha-1} u(t)$ are said to be equiconvergent at infinity whenever for all $\varepsilon>0$ if there exists $\delta=\delta(\varepsilon)>0$ such that

$$
\left|e^{-t_{1}} u\left(t_{1}\right)-e^{-t_{2}} u\left(t_{2}\right)\right|<\varepsilon \text { and }\left|e^{-t_{1}} D^{\alpha-1} u\left(t_{1}\right)-e^{-t_{2}} D^{\alpha-1} u\left(t_{2}\right)\right|<\varepsilon,
$$

for any $t_{1}, t_{2}>\delta$ and $u \in Z$.

Lemma 3.3. Let $L$ be the operator defined in the above and assume that $(H 0)$ holds. Then

$$
\operatorname{Ker}(L)=\left\{c t^{\alpha-1}, c \in \mathbb{R}\right\} \text { and } \operatorname{Im}(L)=\left\{y \in Y: \sum_{i=1}^{m-2} \beta_{i} \int_{\xi_{i}}^{+\infty} y(s) d s=0\right\} .
$$

Proof. Let $u \in \operatorname{Ker}(L)$. From Lemma 2.9 and $D_{0^{+}}^{\alpha} u(t)=0$, we have

$$
u(t)=c_{1} t^{\alpha-1}+c_{2} t^{\alpha-2}, \text { for some constants } c_{1}, c_{2} \in \mathbb{R} .
$$

Using the condition $I_{0^{+}}^{2-\alpha} u(0)=0$, we get $c_{2}=0$. Hence $u(t)=c_{1} t^{\alpha-1}$. If $y \in \operatorname{Im}(L)$, then there exists $u \in D(L)$ such that $D_{0^{+}}^{\alpha} u(t)=y(t)$. Hence

$$
u(t)=I_{0^{+}}^{\alpha} y(t)+c_{1} t^{\alpha-1}+c_{2} t^{\alpha-2}, \text { for some constants } c_{1}, c_{2} \in \mathbb{R} .
$$

From $I_{0^{+}}^{2-\alpha} u(0)=0$, we have $c_{2}=0$. In addition,

$$
\begin{aligned}
D_{0^{+}}^{\alpha-1} u(t) & =D_{0^{+}}^{\alpha-1} I_{0^{+}}^{\alpha} y(t)+c_{1} D_{0^{+}}^{\alpha-1}\left(t^{\alpha-1}\right) \\
& =I_{0^{+}}^{1} y(t)+c_{1} \Gamma(\alpha) \\
& =\int_{0}^{t} y(s) d s+c_{1} \Gamma(\alpha) .
\end{aligned}
$$

So

$$
\lim _{t \rightarrow+\infty} D_{0^{+}}^{\alpha-1} u(t)=\int_{0}^{+\infty} y(s) d s+c_{1} \Gamma(\alpha)
$$

and

$$
D_{0^{+}}^{\alpha-1} u\left(\xi_{i}\right)=\int_{0}^{\xi_{i}} y(s) d s+c_{1} \Gamma(\alpha)
$$

From

$$
\lim _{t \rightarrow+\infty} D_{0^{+}}^{\alpha-1} u(t)=\sum_{i=1}^{m-2} \beta_{i} D_{0^{+}}^{\alpha-1} u\left(\xi_{i}\right)
$$

and condition $(H 0)$, we get

$$
\int_{0}^{+\infty} y(s) d s=\sum_{i=1}^{m-2} \beta_{i} \int_{0}^{\xi_{i}} y(s) d s
$$

Hence

$$
\sum_{i=1}^{m-2} \beta_{i} \int_{\xi_{i}}^{+\infty} y(s) d s=0
$$

Lemma 3.4. The operator $L: D(L) \subset X \rightarrow Y$ is a Fredholm operator of index zero. Furthermore, the linear continuous projectors $P: X \rightarrow X$ and $Q: Y \rightarrow Y$ satisfy

$$
(P u)(t)=\frac{D_{0^{+}}^{\alpha-1} u(0)}{\Gamma(\alpha)} t^{\alpha-1} \text { and }(Q y)(t)=\frac{e^{-t}}{\sum_{i=1}^{m-2} \beta_{i} e^{-\xi_{i}}} \sum_{i=1}^{m-2} \beta_{i} \int_{\xi_{i}}^{+\infty} y(s) d s .
$$


Proof. For all $u \in X$ and $t \in[0,+\infty)$, we have

$$
\begin{aligned}
P(P u)(t) & =\frac{D_{0^{+}}^{\alpha-1}(P u)(0)}{\Gamma(\alpha)} t^{\alpha-1} \\
& =\frac{\frac{D_{0^{+}}^{\alpha-1} u(0)}{\Gamma(\alpha)}}{\Gamma(\alpha)} \Gamma(\alpha) t^{\alpha-1} \\
& =(P u)(t) .
\end{aligned}
$$

For all $y \in Y$ and $t \in[0,+\infty)$, we get

$$
\begin{aligned}
Q(Q y)(t) & =\frac{e^{-t}}{\sum_{i=1}^{m-2} \beta_{i} e^{-\xi_{i}}} \sum_{i=1}^{m-2} \beta_{i} \int_{\xi_{i}}^{+\infty}(Q y)(s) d s \\
& =\frac{e^{-t}}{\sum_{i=1}^{m-2} \beta_{i} e^{-\xi_{i}}}\left(\sum_{i=1}^{m-2} \beta_{i} \int_{\xi_{i}}^{+\infty} y(s) d s\right)\left(\frac{1}{\sum_{i=1}^{m-2} \beta_{i} e^{-\xi_{i}}} \sum_{i=1}^{m-2} \beta_{i} \int_{\xi_{i}}^{+\infty} e^{-s} d s\right) \\
& =(Q y)(t) .
\end{aligned}
$$

On the other hand, we have $\operatorname{Ker}(Q)=\operatorname{Im}(L)$. Indeed, $(Q y)(t)=0$ is equivalent to

$$
\sum_{i=1}^{m-2} \beta_{i} \int_{\xi_{i}}^{+\infty} y(s) d s=0
$$

i.e., $y \in \operatorname{Im}(L)$. For all $y \in Y, y_{1}=y-Q y \in \operatorname{Im}(L)$. Hence $Y=\operatorname{Im}(L)+\operatorname{Im}(Q)$. Since $\operatorname{Im}(L) \cap \operatorname{Im}(Q)=$ $\{0\}$, we have $Y=\operatorname{Im}(L) \oplus \operatorname{Im}(Q)$. It follows that

$$
\operatorname{dimKer}(L)=1=\operatorname{dimIm}(Q)=\operatorname{codimIm}(L) .
$$

So $L$ is a Fredholm operator of index zero. Furthermore, since $\operatorname{Im}(P)=\operatorname{Ker}(L), P^{2} u=P u$ and $u=$ $(u-P u)+P u$, we have $X=\operatorname{Ker}(L)+\operatorname{Ker}(P)$. Finally $\operatorname{Ker}(L) \cap \operatorname{Ker}(P)=\{0\}$ implies that $X=\operatorname{Ker}(L) \oplus$ $\operatorname{Ker}(P)$.

Lemma 3.5. Let $L_{P}=\left.L\right|_{D(L) \cap \operatorname{Ker}(P)}: D(L) \cap \operatorname{Ker}(P) \rightarrow \operatorname{Im}(L)$. The inverse $K_{P}$ of $L_{P}$ is given by

$$
\left(K_{P} y\right)(t)=I_{0^{+}}^{\alpha} y(t), y \in \operatorname{Im}(L) .
$$

Then

$$
\left\|K_{P} y\right\|_{X} \leq \frac{1}{\Gamma(\alpha)}\|y\|_{1}
$$

Proof. For $y \in \operatorname{Im}(L)$, we have

$$
\left(L_{P} K_{P}\right) y(t)=D_{0^{+}}^{\alpha}\left(K_{P} y(t)\right)=D_{0^{+}}^{\alpha} I_{0^{+}}^{\alpha} y(t)=y(t)
$$

and for $u \in D(L) \cap \operatorname{Ker}(P)$, we have

$$
\left(K_{P} L_{P}\right) u(t)=\left(K_{P}\right) D_{0^{+}}^{\alpha} u(t)=I_{0^{+}}^{\alpha} D_{0^{+}}^{\alpha} u(t)=u(t)+c_{1} t^{\alpha-1}+c_{2} t^{\alpha-2} .
$$

Since $u \in D(L) \cap \operatorname{Ker}(P)$, we have

$$
\left(K_{P} L_{P}\right) u \in D(L) \cap \operatorname{Ker}(P) .
$$

Hence $I_{0^{+}}^{2-\alpha} u(0)=0, I_{0^{+}}^{2-\alpha}\left(\left(K_{P} L_{P}\right) u(0)\right)=0$. In addition,

$$
I_{0^{+}}^{2-\alpha}\left(\left(K_{P} L_{P}\right) u(t)\right)=I_{0^{+}}^{2-\alpha} u(t)+c_{1} \Gamma(\alpha) t+c_{2} \Gamma(\alpha-1)
$$


implies that $c_{2}=0$. On the other hand, $P u(t)=0, P\left(\left(K_{P} L_{P}\right) u(t)\right)=0$ and

$$
P\left(\left(K_{P} L_{P}\right) u(t)\right)=P u(t)+c_{1} t^{\alpha-1}
$$

imply $c_{1}=0$ and $\left(K_{P} L_{P}\right) u(t)=u(t)$. This shows that $K_{P}=\left(\left.L\right|_{D(L) \cap \operatorname{Ker}(P)}\right)^{-1}$. Moreover, we have the estimates:

$$
\begin{aligned}
e^{-t}\left|\left(K_{P} y\right)(t)\right| & \leq \frac{e^{-t}}{\Gamma(\alpha)} \int_{0}^{t}(t-s)^{\alpha-1}|y(s)| d s \\
& \leq \frac{e^{-t} t^{\alpha-1}}{\Gamma(\alpha)} \int_{0}^{+\infty}|y(s)| d s \\
& \leq \sup _{t \geq 0}\left(\frac{e^{-t} t^{\alpha-1}}{\Gamma(\alpha)}\right)\|y\|_{1} \\
& \leq \frac{\left(e^{-1}(\alpha-1)\right)^{\alpha-1}}{\Gamma(\alpha)}\|y\|_{1} \\
& \leq \frac{1}{\Gamma(\alpha)}\|y\|_{1}
\end{aligned}
$$

and

$$
\begin{aligned}
e^{-t}\left|D_{0^{+}}^{\alpha-1}\left(K_{P} y\right)(t)\right| & =e^{-t}\left|I_{0^{+}}^{1} y(t)\right| \\
& \leq \int_{0}^{t}|y(s)| d s \\
& \leq \frac{1}{\Gamma(\alpha)}\|y\|_{1} .
\end{aligned}
$$

This implies that

$$
\left\|K_{P} y\right\|_{X} \leq \frac{1}{\Gamma(\alpha)}\|y\|_{1}
$$

Lemma 3.6. Let $(H 0)-(H 2)$ hold and suppose that $\Omega$ is an open bounded subset of $X$ such that $D(L) \cap$ $\bar{\Omega} \neq \emptyset$. Then $N$ is L-compact on $\bar{\Omega}$.

Proof. The proof is split into three parts.

Claim 1. $K_{P}(I-Q) N(\bar{\Omega})$ is uniformly bounded. Assume that $\Omega=B(O, r)$. For any $u \in \bar{\Omega},\|u\|_{X} \leq r$. From $(H 0)-(H 2)$ and for $u \in \bar{\Omega}$, we have

$$
\begin{aligned}
|Q N u| & \leq \frac{e^{-t}}{\sum_{i=1}^{m-2} \beta_{i} e^{-\xi_{i}}} \sum_{i=1}^{m-2} \beta_{i} \int_{\xi_{i}}^{+\infty}\left|f\left(s, u(s), D_{0^{+}}^{\alpha-1} u(s)\right)\right| d s \\
& \leq \frac{e^{-t}}{\sum_{i=1}^{m-2} \beta_{i} e^{-\xi_{i}}} \sum_{i=1}^{m-2} \beta_{i} \int_{0}^{+\infty}\left|f\left(s, u(s), D_{0^{+}}^{\alpha-1} u(s)\right)\right| d s \\
& \leq \frac{1}{\sum_{i=1}^{m-2} \beta_{i} e^{-\xi_{i}}} \int_{0}^{+\infty}\left(e^{s} a(s)\|u\|_{X}+e^{s} b(s)\|u\|_{X}+c(s)\right) d s \\
& \left.\leq \frac{1}{\sum_{i=1}^{m-2} \beta_{i} e^{-\xi_{i}}}\left(r \int_{0}^{+\infty} e^{s}(a(s)+b(s)) d s+\int_{0}^{+\infty} c(s)\right) d s\right) .
\end{aligned}
$$

Thus

$$
\left.\|Q N u\|_{1} \leq \frac{1}{\sum_{i=1}^{m-2} \beta_{i} e^{-\xi_{i}}}\left(r \int_{0}^{+\infty} e^{s}(a(s)+b(s)) d s+\int_{0}^{+\infty} c(s)\right) d s\right)
$$


This implies that $Q N(\bar{\Omega})$ is bounded. Next, we show that $K_{P}(I-Q) N(\bar{\Omega})$ is compact. For $u \in \bar{\Omega}$, we have

$$
\begin{aligned}
\|N u\|_{1} & \leq \int_{0}^{+\infty}\left|f\left(s, u(s), D_{0^{+}}^{\alpha-1} u(s)\right)\right| d s \\
& \left.\leq r \int_{0}^{+\infty} e^{s}(a(s)+b(s)) d s+\int_{0}^{+\infty} c(s)\right) d s
\end{aligned}
$$

From Lemma 3.5, we get

$$
\begin{aligned}
\left\|K_{P}(I-Q) N u\right\|_{X} \leq & \frac{1}{\Gamma(\alpha)}\|(I-Q) N u\|_{1} \\
\leq & \frac{1}{\Gamma(\alpha)}\left(\|N u\|_{1}+\|Q N u\|_{1}\right) \\
\leq & \frac{1}{\Gamma(\alpha)}\left(r \int_{0}^{+\infty} e^{s}(a(s)+b(s)) d s+\int_{0}^{+\infty} c(s) d s\right. \\
& \left.+\frac{1}{\sum_{i=1}^{m-2} \beta_{i} e^{-\xi}}\left(r \int_{0}^{+\infty} e^{s}(a(s)+b(s)) d s+\int_{0}^{+\infty} c(s) d s\right)\right) \\
\leq & \frac{1}{\Gamma(\alpha)}\left(1+\frac{1}{\sum_{i=1}^{m-2} \beta_{i} e^{-\xi_{i}}}\right)\left(r \int_{0}^{+\infty} e^{s}(a(s)+b(s)) d s+\int_{0}^{+\infty} c(s) d s\right) .
\end{aligned}
$$

It follows that $K_{P}(I-Q) N(\bar{\Omega})$ is uniformly bounded.

Claim 2. $K_{P}(I-Q) N(\bar{\Omega})$ is equicontinuous. For any $u \in \bar{\Omega}$ and any $t_{1}, t_{2} \in[0, d], t_{1}<t_{2}$ with $d>0$, we have

$$
\begin{aligned}
\mid e^{-t_{1}}\left(K_{P}(I-Q) N u\right)\left(t_{1}\right)- & e^{-t_{2}}\left(K_{P}(I-Q) N u\right)\left(t_{2}\right) \mid \\
= & \frac{1}{\Gamma(\alpha)} \mid \int_{0}^{t_{1}} e^{-t_{1}}\left(t_{1}-s\right)^{\alpha-1}(I-Q) N u(s) d s \\
& -\int_{0}^{t_{2}} e^{-t_{2}}\left(t_{2}-s\right)^{\alpha-1}(I-Q) N u(s) d s \mid \\
= & \frac{1}{\Gamma(\alpha)} \mid \int_{0}^{t_{1}} e^{-t_{1}}\left(t_{1}-s\right)^{\alpha-1}(I-Q) N u(s) d s \\
& +\int_{0}^{t_{1}} e^{-t_{2}}\left(t_{2}-s\right)^{\alpha-1}(I-Q) N u(s) d s \\
& -\int_{0}^{t_{1}} e^{-t_{2}}\left(t_{2}-s\right)^{\alpha-1}(I-Q) N u(s) d s \\
& -\int_{0}^{t_{2}} e^{-t_{2}}\left(t_{2}-s\right)^{\alpha-1}(I-Q) N u(s) d s \mid .
\end{aligned}
$$

\section{Moreover}

$$
\begin{aligned}
\mid e^{-t_{1}}\left(K_{P}(I-Q) N u\right)\left(t_{1}\right)= & e^{-t_{2}}\left(K_{P}(I-Q) N u\right)\left(t_{2}\right) \mid \\
\leq & \frac{1}{\Gamma(\alpha)}\left(\int_{0}^{t_{1}}\left(e^{-t_{2}}\left(t_{2}-s\right)^{\alpha-1}-e^{-t_{1}}\left(t_{1}-s\right)^{\alpha-1}\right)|(I-Q) N u(s)| d s\right. \\
& \left.+\int_{t_{1}}^{t_{2}} e^{-t_{2}}\left(t_{2}-s\right)^{\alpha-1}|(I-Q) N u(s)| d s\right) \\
\leq & \frac{1}{\Gamma(\alpha)}\left(\int_{0}^{t_{1}} e^{-t_{1}}\left(\left(t_{2}-s\right)^{\alpha-1}-\left(t_{1}-s\right)^{\alpha-1}\right)|(I-Q) N u(s)| d s\right. \\
& \left.+e^{-t_{2}} t_{2}^{\alpha-1} \int_{t_{1}}^{t_{2}}|(I-Q) N u(s)| d s\right) \\
\leq & \frac{1}{\Gamma(\alpha)}\left(1+\frac{1}{\sum_{i=1}^{m-2} \beta_{i} e^{-\xi_{i}}}\right)\left(r \int_{0}^{+\infty} e^{s}(a(s)+b(s)) d s\right. \\
& \left.+\int_{0}^{+\infty} c(s) d s\right)\left(\int_{0}^{t_{1}} e^{-t_{1}}\left(\left(t_{2}-s\right)^{\alpha-1}-\left(t_{1}-s\right)^{\alpha-1}\right) d s\right. \\
& \left.+e^{-t_{2}} t_{2}^{\alpha-1} \int_{t_{1}}^{t_{2}} d s\right)
\end{aligned}
$$


which tends to 0 as $t_{2}-t_{1} \rightarrow 0$. On the other hand, we have

$$
\begin{aligned}
\mid e^{-t_{1}} D_{0^{+}}^{\alpha-1}\left(K_{P}(I-Q) N u\right)\left(t_{1}\right)= & e^{-t_{2}} D_{0^{+}}^{\alpha-1}\left(K_{P}(I-Q) N u\right)\left(t_{2}\right) \mid \\
= & \left|e^{-t_{1}} \int_{0}^{t_{1}}(I-Q) N u(s) d s-e^{-t_{2}} \int_{0}^{t_{2}}(I-Q) N u(s) d s\right| \\
\leq & \left(e^{-t_{1}}-e^{-t_{2}}\right) \int_{0}^{t_{1}}|(I-Q) N u(s)| d s \\
& +e^{-t_{2}} \int_{t_{1}}^{t_{2}}|(I-Q) N u(s)| d s \\
\leq & \left(1+\frac{1}{\sum_{i=1}^{m-2} \beta_{i} e^{-\xi_{i}}}\right)\left(r \int_{0}^{+\infty} e^{s}(a(s)+b(s)) d s\right. \\
& \left.+\int_{0}^{+\infty} c(s) d s\right)\left(t_{1}\left(e^{-t_{1}}-e^{-t_{2}}\right)+\left(t_{2}-t_{1}\right) e^{-t_{2}}\right) .
\end{aligned}
$$

The last term tends to 0 as $t_{2}-t_{1} \rightarrow 0$. Hence $K_{P}(I-Q) N(\bar{\Omega})$ is equicontinuous on every compact subinterval of $[0,+\infty)$.

Claim 3. Functions from $\left\{e^{-t} K_{P}(I-Q) N(\bar{\Omega})\right\}$ and functions from $\left\{e^{-t} D_{0^{+}}^{\alpha-1}\left(K_{P}(I-Q) N\right)(\bar{\Omega})\right\}$ are equiconvergent at infinity.

For any $u \in \bar{\Omega}$, we have

$$
\int_{0}^{+\infty}\left|f\left(s, u(s), D^{\alpha-1} u(s)\right)\right| d s<+\infty
$$

With condition $(H 2)$ in mind, for given $\varepsilon>0$, there exists a constant $L>0$ such that

$$
\int_{L}^{+\infty}\left|f\left(s, u(s), D^{\alpha-1} u(s)\right)\right| d s<\varepsilon .
$$

On the other hand, since $\lim _{t \rightarrow+\infty} e^{-t} t^{\gamma}=0,(\gamma \geq 1)$, we find that there exists a constant $\delta>0$ such that, for any $t_{1}, t_{2}>\delta$,

$$
e^{-t_{i}} t_{i}^{\gamma}<\frac{\varepsilon}{2},(i=1,2)
$$

Then, for any $t_{1}, t_{2}>\delta, t_{1}<t_{2}$,

$$
\begin{aligned}
\mid e^{-t_{1}}\left(K_{P}(I-Q) N u\right)\left(t_{1}\right)= & e^{-t_{2}}\left(K_{P}(I-Q) N u\right)\left(t_{2}\right) \mid \\
= & \frac{1}{\Gamma(\alpha)} \mid \int_{0}^{t_{1}} e^{-t_{1}}\left(t_{1}-s\right)^{\alpha-1}(I-Q) N u(s) d s \\
& -\int_{0}^{t_{2}} e^{-t_{2}}\left(t_{2}-s\right)^{\alpha-1}(I-Q) N u(s) d s \mid \\
\leq & \frac{1}{\Gamma(\alpha)}\left(\int_{0}^{t_{1}} e^{-t_{1}} t_{1}^{\alpha-1}|(I-Q) N u(s)| d s\right. \\
& \left.+\int_{0}^{t_{2}} e^{-t_{2}} t_{2}^{\alpha-1}|(I-Q) N u(s)| d s\right) \\
\leq & \frac{1}{\Gamma(\alpha)}\left(1+\frac{1}{\sum_{i=1}^{m-2} \beta_{i} e^{-\xi_{i}}}\right)\left(\int_{0}^{+\infty}\left|f\left(s, u(s), D^{\alpha-1} u(s)\right)\right| d s\right) \\
& \times\left(\int_{0}^{t_{1}} e^{-t_{1}} t_{1}^{\alpha-1} d s+\int_{0}^{t_{2}} e^{-t_{2}} t_{2}^{\alpha-1} d s\right) \\
\leq & \frac{1}{\Gamma(\alpha)}\left(1+\frac{1}{\sum_{i=1}^{m-2} \beta_{i} e^{-\xi_{i}}}\right)\left(\int_{0}^{L}\left|f\left(s, u(s), D^{\alpha-1} u(s)\right)\right| d s\right. \\
& \left.+\int_{L}^{+\infty}\left|f\left(s, u(s), D^{\alpha-1} u(s)\right)\right| d s\right)\left(e^{-t_{1}} t_{1}^{\alpha}+e^{-t_{2}} t_{2}^{\alpha}\right) \\
\leq & \frac{1}{\Gamma(\alpha)}\left(1+\frac{1}{\sum_{i=1}^{m-2} \beta_{i} e^{-\xi_{i}}}\right) \\
& \times\left(\max _{s \in[0, L], u \in \bar{\Omega}}\left|f\left(s, u(s), D^{\alpha-1} u(s)\right)\right| L+\varepsilon\right) \varepsilon .
\end{aligned}
$$


Moreover

$$
\begin{aligned}
\mid e^{-t_{1}} D_{0^{+}}^{\alpha-1}\left(K_{P}(I-Q) N u\right)\left(t_{1}\right)= & e^{-t_{2}} D_{0^{+}}^{\alpha-1}\left(K_{P}(I-Q) N u\right)\left(t_{2}\right) \mid \\
= & \left|e^{-t_{1}} \int_{0}^{t_{1}}(I-Q) N u(s) d s-e^{-t_{2}} \int_{0}^{t_{2}}(I-Q) N u(s) d s\right| \\
\leq & e^{-t_{1}} \int_{0}^{t_{1}}|(I-Q) N u(s)| d s+e^{-t_{2}} \int_{0}^{t_{2}}|(I-Q) N u(s)| d s \\
\leq & \left(1+\frac{1}{\sum_{i=1}^{m-2} \beta_{i} e^{-\xi_{i}}}\right)\left(\int_{0}^{+\infty}\left|f\left(s, u(s), D^{\alpha-1} u(s)\right)\right| d s\right) \\
& \times\left(t_{1} e^{-t_{1}}+t_{2} e^{-t_{2}}\right) \\
\leq & \left(1+\frac{1}{\sum_{i=1}^{m-2} \beta_{i} e^{-\xi_{i}}}\right)\left(\int_{0}^{L}\left|f\left(s, u(s), D^{\alpha-1} u(s)\right)\right| d s\right. \\
& \left.+\int_{L}^{+\infty}\left|f\left(s, u(s), D^{\alpha-1} u(s)\right)\right| d s\right) \times\left(t_{1} e^{-t_{1}}+t_{2} e^{-t_{2}}\right) \\
\leq & \left(1+\frac{1}{\sum_{i=1}^{m-2} \beta_{i} e^{-\xi_{i}}}\right) \\
& \times\left(\max _{s \in[0, L], u \in \Omega}\left|f\left(s, u(s), D^{\alpha-1} u(s)\right)\right| L+\varepsilon\right) \varepsilon . \\
&
\end{aligned}
$$

We deduce that the functions from $\left\{e^{-t} K_{P}(I-Q) N(\bar{\Omega})\right\}$ and functions from $\left\{e^{-t} D_{0^{+}}^{\alpha-1}\left(K_{P}(I-Q) N\right)(\bar{\Omega})\right\}$ are equiconvergent at infinity. By Lemma 3.1, we conclude that $K_{P}(I-Q) N(\bar{\Omega})$ is relatively compact. This ends the proof of the Lemma.

\section{The Main Result}

Theorem 4.1. Assume that conditions $(H 0)-(H 4)$ are satisfied. Then problem (1.1) has at least one solution.

Proof. The proof is split into three parts.

Claim 1. Consider the set

$$
\Omega_{1}=\{u \in D(L) \backslash \operatorname{Ker}(L): L u=\lambda N u, \lambda \in(0,1)\} .
$$

For $u \in \Omega_{1}$ and $\lambda \neq 0$, we have $N u=L\left(\frac{1}{\lambda} u\right) \in \operatorname{Im}(L)=\operatorname{Ker}(Q)$. Hence $Q N u=0$, which implies

$$
\sum_{i=1}^{m-2} \beta_{i} \int_{\xi_{i}}^{+\infty} f\left(s, u(s), D_{0^{+}}^{\alpha-1} u(s)\right) d s=0
$$

By condition $(H 3)$, there exists $t_{0} \in[0,+\infty)$ such that $\left|D_{0^{+}}^{\alpha-1} u\left(t_{0}\right)\right| \leq M_{1}$. Since

$$
D_{0^{+}}^{\alpha-1} u(0)=D_{0^{+}}^{\alpha-1} u\left(t_{0}\right)-\int_{0}^{t_{0}} D_{0^{+}}^{\alpha} u(s) d s,
$$

we have

$$
\begin{aligned}
\left|D_{0^{+}}^{\alpha-1} u(0)\right| & \leq\left|D_{0^{+}}^{\alpha-1} u\left(t_{0}\right)\right|+\int_{0}^{t_{0}}\left|D_{0^{+}}^{\alpha} u(s)\right| d s \\
& \leq M_{1}+\int_{0}^{+\infty}|L u(s)| d s \\
& \leq M_{1}+\int_{0}^{+\infty}|N u(s)| d s \\
& \leq M_{1}+\|N u\|_{1} .
\end{aligned}
$$


Moreover, for $u \in \Omega_{1}, u \in D(L) \backslash \operatorname{Ker}(L)$, we have $(I-P) u \in D(L) \cap \operatorname{Ker}(P)$ and $L_{P} P u=0$. Using (H2), Lemma 3.5 and $u=P u+(I-P) u=P u+K_{P} L_{P}(I-P) u$, we get

$$
\begin{aligned}
\|u\|_{X} & \leq\|P u\|_{X}+\left\|K_{P} L_{P}(I-P) u\right\|_{X} \\
& \leq \frac{1}{\Gamma(\alpha)}\left|D_{0^{+}}^{\alpha-1} u(0)\right|+\frac{1}{\Gamma(\alpha)}\left\|L_{P}(I-P) u\right\|_{1} \\
& \leq \frac{1}{\Gamma(\alpha)}\left(M_{1}+\|N u\|_{1}\right)+\frac{1}{\Gamma(\alpha)}\left\|L_{P} u\right\|_{1} \\
& \leq \frac{1}{\Gamma(\alpha)}\left(M_{1}+\|N u\|_{1}\right)+\frac{1}{\Gamma(\alpha)}\|N u\|_{1} \\
& \leq \frac{1}{\Gamma(\alpha)} M_{1}+\frac{2}{\Gamma(\alpha)}\|N u\|_{1} \\
& \leq \frac{1}{\Gamma(\alpha)} M_{1}+\frac{2}{\Gamma(\alpha)}\left(\|u\|_{X} \int_{0}^{+\infty} e^{s}(a(s)+b(s)) d s+\int_{0}^{+\infty} c(s) d s\right)
\end{aligned}
$$

Hence

$$
\|u\|_{X} \leq \frac{1}{1-\frac{2}{\Gamma(\alpha)} \int_{0}^{+\infty} e^{s}(a(s)+b(s)) d s}\left(\frac{M_{1}}{\Gamma(\alpha)}+\frac{2}{\Gamma(\alpha)}\|c\|_{1}\right) .
$$

Therefore, $\Omega_{1}$ is bounded.

\section{Claim 2. Let}

$$
\Omega_{2}=\{u \in \operatorname{Ker}(L): N u \in \operatorname{Im}(L)\} .
$$

For $u \in \Omega_{2}$, we have $u \in \operatorname{Ker}(L)$, i.e., $u(t)=c t^{\alpha-1}, c \in \mathbb{R}$ and $Q N u=0$. Hence

$$
\sum_{i=1}^{m-2} \beta_{i} \int_{\xi_{i}}^{+\infty} f\left(s, u(s), D_{0^{+}}^{\alpha-1} u(s)\right) d s=0 .
$$

From $(H 3)$, there exists $t_{1} \in[0,+\infty)$ such that $\left|D_{0^{+}}^{\alpha-1} u\left(t_{1}\right)\right| \leq M_{1}$, which implies $|c| \leq \frac{M_{1}}{\Gamma(\alpha)}$. It follows that

$$
\|u\|_{X} \leq \frac{M_{1}}{\Gamma(\alpha)}
$$

This shows that $\Omega_{2}$ is bounded in $X$.

\section{Claim 3. Let}

$$
\Omega_{3}=\{u \in \operatorname{Ker}(L):-\lambda J u+(1-\lambda) Q N u=0, \lambda \in[0,1]\},
$$

where $J: \operatorname{Ker}(L) \rightarrow \operatorname{Im}(Q)$ is the linear isomorphism given by $J\left(c t^{\alpha-1}\right)=c e^{-t}$ for all $c \in \mathbb{R}, t \geq 0$. For $u \in \Omega_{3}$, we have

$$
u(t)=c_{0} t^{\alpha-1} \text { and } \lambda c_{0}=(1-\lambda) \frac{\sum_{i=1}^{m-2} \beta_{i}}{\sum_{i=1}^{m-2} \beta_{i} e^{-\xi_{i}}} \int_{\xi_{i}}^{+\infty} f\left(s, c_{0} t^{\alpha-1}, c_{0} \Gamma(\alpha)\right) d s .
$$

If $\lambda=0$, then

$$
\sum_{i=1}^{m-2} \beta_{i} \int_{\xi_{i}}^{+\infty} f\left(s, c_{0} t^{\alpha-1}, c_{0} \Gamma(\alpha)\right) d s=0 .
$$

By $(H 3)$, there exists $t_{1} \geq 0$ such that $\left|D_{0^{+}}^{\alpha-1} u\left(t_{1}\right)\right| \leq M_{1}$, i.e., $\left|c_{0}\right| \leq \frac{M_{1}}{\Gamma(\alpha)}$ and $\|u\|_{X} \leq \frac{M_{1}}{\Gamma(\alpha)}$. If $\lambda=1$, then $u=0$. If $0<\lambda<1$ and $\left|c_{0}\right|>M_{2}$, we suppose that, in (H4),

$$
c_{0} \frac{\sum_{i=1}^{m-2} \beta_{i}}{\sum_{i=1}^{m-2} \beta_{i} e^{-\xi_{i}}} \int_{\xi_{i}}^{+\infty} f\left(s, c_{0} t^{\alpha-1}, c_{0} \Gamma(\alpha)\right) d s<0 .
$$


Hence,

$$
\lambda c_{0}^{2}=(1-\lambda) c_{0} \frac{\sum_{i=1}^{m-2} \beta_{i}}{\sum_{i=1}^{m-2} \beta_{i} e^{-\xi_{i}}} \int_{\xi_{i}}^{+\infty} f\left(s, c_{0} t^{\alpha-1}, c_{0} \Gamma(\alpha)\right) d s<0 .
$$

This is a contradiction to $\lambda c_{0}^{2} \geq 0$. So $\left|c_{0}\right| \leq M_{2}$ and $\|u\|_{X} \leq M_{2}$. This proves that $\Omega_{3}$ is bounded. Likewise, we can prove that the following set

$$
\Omega_{3}=\{u \in \operatorname{Ker}(L): \lambda J u+(1-\lambda) Q N u=0, \lambda \in[0,1]\} .
$$

is bounded.

Next, we prove that all conditions of Theorem 2.3 are satisfied. Let $\Omega$ be an open bounded subset of $X$ such that $\sqcup_{i=1}^{i=3} \bar{\Omega}_{i} \subset \Omega$. From Lemma 3.4, we known that $L$ is a Fredholm operator of index zero. By Lemma 3.6, we have $N$ is $L$-compact on $\bar{\Omega}$. Since $\Omega_{i},(i=1,2,3)$ are bounded sets and $\Omega_{i} \subset \Omega$, we have

$$
\text { (1) } L u \neq \lambda N u \text { for all }(u, \lambda) \in[(D(L) \backslash \operatorname{Ker}(L)) \cap \partial \Omega] \times(0,1),
$$

(2) $N u \notin \operatorname{Im}(L)$ for all $u \in \operatorname{Ker}(L) \cap \partial \Omega$.

First we prove that condition (3) of Theorem 2.3 is satisfied. Let

$$
H(u, \lambda)= \pm \lambda J u+(1-\lambda) Q N u .
$$

As $\bar{\Omega}_{3} \subset \Omega$, for all $u \in \operatorname{Ker}(L) \cap \partial \Omega$ and $\lambda \in[0,1]$, we obtain that $H(u, \lambda) \neq 0$. By the homotopy property of the degree, we obtain

$$
\begin{aligned}
\operatorname{deg}\left(\left.Q N\right|_{\operatorname{Ker}(L)}, \operatorname{Ker}(L) \cap \Omega, 0\right) & =\operatorname{deg}(H(., 0), \operatorname{Ker}(L) \cap \Omega, 0) \\
& =\operatorname{deg}(H(., 1), \operatorname{Ker}(L) \cap \Omega, 0) \\
& =\operatorname{deg}( \pm J, \operatorname{Ker}(L) \cap \Omega, 0) \neq 0 .
\end{aligned}
$$

So condition (3) of Theorem 2.3 is verified. We conclude that $L u=N u$ has at least one solution in $D(L) \cap \bar{\Omega}$, i.e., problem (1.1) has at least one solution in $X$. This ends the proof of Theorem 4.1.

\section{AN EXAMPLE}

Example 5.1. Consider the 4-point BVP:

$$
\left\{\begin{array}{l}
D_{0^{+}}^{\frac{3}{2}} u(t)=\frac{1}{(1+t)^{2}} \sin (u(t))+\frac{D_{0^{+}}^{\frac{1}{2}} u(t)}{3 e^{2 t}}+2 e^{-t}, \quad t \in(0,+\infty), \\
I_{0^{+}}^{\frac{1}{2}} u(0)=0, \quad \lim _{t \rightarrow+\infty} D_{0^{+}}^{\frac{1}{2}} u(t)=\frac{1}{4} D_{0^{+}}^{\frac{1}{2}} u(1)+\frac{3}{4} D_{0^{+}}^{\frac{1}{2}} u(2) .
\end{array}\right.
$$

Then (5.1) has at least one solution in the space:

$$
X=\left\{u: u, D_{0^{+}}^{\frac{1}{2}} u \in C([0,+\infty), \mathbb{R}): \lim _{t \rightarrow+\infty} e^{-t}|u(t)| \text { and } \lim _{t \rightarrow+\infty} e^{-t}\left|D_{0^{+}}^{\frac{1}{2}} u(t)\right| \text { exist }\right\} .
$$

Here we have $\alpha=\frac{3}{2}, \Gamma\left(\frac{3}{2}\right)=\frac{\sqrt{\pi}}{2}, \beta_{1}=\frac{1}{4}, \beta_{2}=\frac{3}{4}, \xi_{1}=1$ and $\xi_{2}=2$. Let

$$
f(t, u, v)=\frac{1}{(1+t)^{2}} \sin u+\frac{v}{3 e^{2 t}}+2 e^{-t} .
$$

Then

$(H 0)$ is satisfied for $\beta_{1}+\beta_{2}=1$. 
(H1) $f:[0,+\infty) \times \mathbb{R} \times \mathbb{R} \rightarrow \mathbb{R}$ is continuous.

(H2) $|f(t, u, v)| \leq \frac{1}{3 e^{2 t}}|v|+\frac{1}{(1+t)^{2}}+2 e^{-t}$. So we may take

$$
a(t)=0, b(t)=\frac{1}{3 e^{2 t}}, c(t)=\frac{1}{(1+t)^{2}}+2 e^{-t}
$$

and note that $e^{t} a(t), e^{t} b(t)$ and $c(t) \in L^{1}[0,+\infty)$ verify

$$
\frac{2}{\Gamma(\alpha)} \int_{0}^{+\infty} e^{s}(a(s)+b(s)) d s=\frac{4}{3 \sqrt{\pi}}<1 .
$$

(H3) Let $M_{1}=1$. If $\left|D_{0^{+}}^{\frac{1}{2}} u(t)\right|>1, t \geq 0$. Then

$$
\begin{aligned}
\beta_{1} \int_{\xi_{1}}^{+\infty} f\left(s, u(s), D_{0^{+}}^{\frac{1}{2}} u(s)\right) d s+ & \beta_{2} \int_{\xi_{2}}^{+\infty} f\left(s, u(s), D_{0^{+}}^{\frac{1}{2}} u(s)\right) d s \\
> & \frac{1}{4} \int_{1}^{+\infty}\left(-\frac{1}{(1+s)^{2}}+\frac{1}{3} e^{-2 s}+2 e^{-s}\right) d s \\
& +\frac{3}{4} \int_{2}^{+\infty}\left(-\frac{1}{(1+s)^{2}}+\frac{1}{3} e^{-2 s}+2 e^{-s}\right) d s \\
\simeq & 0.01987 \neq 0 .
\end{aligned}
$$

(H4) Let $M_{2}=150$. If $u(t)=c \sqrt{t}, t \geq 0$ with $|c|>150$, then

$$
\begin{array}{r}
\frac{\beta_{1}}{\beta_{1} e^{-\xi_{1}}+\beta_{2} e^{-\xi_{2}}} \int_{\xi_{1}}^{+\infty} f\left(s, c \sqrt{s}, \frac{\sqrt{\pi}}{2} c\right) d s+\frac{\beta_{2}}{\beta_{1} e^{-\xi_{1}}+\beta_{2} e^{-\xi_{2}}} \int_{\xi_{2}}^{+\infty} f\left(s, c \sqrt{s}, \frac{\sqrt{\pi}}{2} c\right) d s \\
<\frac{1}{e^{-1}+3 e^{-2}} \int_{1}^{+\infty}\left(\frac{1}{(1+s)^{2}}-\frac{150 \sqrt{\pi}}{6} e^{-2 s}+2 e^{-s}\right) d s \\
+\frac{3}{e^{-1}+3 e^{-2}} \int_{2}^{+\infty}\left(\frac{1}{(1+s)^{2}}-\frac{150 \sqrt{\pi}}{6} e^{-2 s}+2 e^{-s}\right) d s \\
\simeq-1.50933<0 .
\end{array}
$$

Moreover, if $c>0$, then

$$
c\left(\frac{\beta_{1}}{\beta_{1} e^{-\xi_{1}}+\beta_{2} e^{-\xi_{2}}} \int_{\xi_{1}}^{+\infty} f\left(s, c \sqrt{s}, \frac{\sqrt{\pi}}{2} c\right) d s+\frac{\beta_{2}}{\beta_{1} e^{-\xi_{1}}+\beta_{2} e^{-\xi_{2}}} \int_{\xi_{2}}^{+\infty} f\left(s, c \sqrt{s}, \frac{\sqrt{\pi}}{2} c\right) d s\right)<0
$$

or, if $c<0$, then

$$
c\left(\frac{\beta_{1}}{\beta_{1} e^{-\xi_{1}}+\beta_{2} e^{-\xi_{2}}} \int_{\xi_{1}}^{+\infty} f\left(s, c \sqrt{s}, \frac{\sqrt{\pi}}{2} c\right) d s+\frac{\beta_{2}}{\beta_{1} e^{-\xi_{1}}+\beta_{2} e^{-\xi_{2}}} \int_{\xi_{2}}^{+\infty} f\left(s, c \sqrt{s}, \frac{\sqrt{\pi}}{2} c\right) d s\right)>0 .
$$

Hence all conditions of Theorem 4.1 hold, which implies that problem (5.1) has at least one solution in $X$.

\section{REFERENCES}

[1] C. P. Gupta, A second order m-point boundary value problem at resonance, Nonlinear Anal. 24 (1995), 1483-1489.

[2] W. Jiang, B. Wang, Z. Wang, Solvability of second-order multi point boundary value problem at resonance on the half-line with $\operatorname{dim} \operatorname{Ker} L=2$, Electron. J. Differential Equations, 2011 (2011), No. 120.

[3] W. Jiang, C. Yang, The existence of positive solutions for multi-point boundary value problem at resonance on the halfline, Boundary Value Probl. 2016 (2016), Article ID 13.

[4] N. Kosmatov, Multi-point boundary value problems on an unbounded domain at resonance, Nonlinear Anal. 68 (2008), 2158-2171. 
[5] Z. B. Bai, Solvability for a class of fractional $m$-point boundary value problem at resonance, Comput. Math. Appl. 62 (2011), 1292-1302.

[6] J. Appell, B. López, K. Sadarangani, Existence and uniqueness of solutions for a nonlinear fractional initial value problem involving Caputo derivatives, J. Nonlinear Var. Anal. 2 (2018), 25-33.

[7] W. H. Jiang, The existence of solutions for boundary value problems of fractional differential equations at resonance, Nonlinear Anal. 74(2011), 1987-1994.

[8] H. Qu, X. Liu, Existence of nonnegative solutions for a fractional $m$-point boundary value problem at resonance, Boundary Value Probl. 2013 (2013), Article ID 127.

[9] A. Guezane-Lakoud, R. Khaldi, On a boundary value problem at resonance on the half line, J. Fractional Calculus Appl. 8 (2017), 159-167.

[10] H. C. Zhou, C. H. Kou, F. Xie, Existence of solutions for fractional differential equations with multi-point boundary conditions at resonance on a half-line, Electron. J. Qual. Theory Differ. Equ. 2011 (2011), Article ID 27.

[11] Y. Chen, X. Tang, Positive solutions of fractional differential equations at resonance on the half-line, Bound. Value Probl. 2012 (2012), Article ID 64.

[12] Y. Gholami, Existence of an unbounded solution for multi-point boundary value problems of fractional differential equations on an infinite domain, Fractional Differential Calculus, 4 (2014), 125-136.

[13] J. Mawhin, Topological degree methods in nonlinear boundary value problems, CBMS Reg. Conf. Ser. Math., vol. 40, American Mathematical Society, Providence, RI, 1979.

[14] A.A. Kilbas, H.M. Srivastava, J.J. Trujillo, Theory and Applications of Fractional Differential Equations, Elsevier Science B.V., Amsterdam, 2006.

[15] K. Oldham, J. Spanier, The Fractional Calculus, Academic Press, New York, 1974.

[16] X. Su, S. Zhang, Unbounded solutions to a boundary value problem of fractional order on the half-line, Comput. Math. Appl. 61 (2011), 1079-1087. 